\title{
Aprender lengua o no aprender lengua. La adquisición de la competencia comunicativa en Educación Secundaria en un entorno altamente tecnológico. Un estudio desde Cataluña (España)
}

\section{Learning language or not learning language. The acquisition of the communicative competence in secondary education in a highly technological environment. A study from Catalonia (Spain)}

\author{
Juan GONZÁLEZ MARTÍNEZ, Cinta ESPUNY VIDAL y \\ Mercè GISBERT CERVERA \\ Universitat Rovira i Virgili.
}

Recibido: Julio 2013

Aceptado: Noviembre 2013

\begin{abstract}
Resumen
En los últimos tres años, España ha implementado un modelo educativo one-to-one, basado en una presencia constante y permanente de la tecnología en las aulas a lo largo de los últimos cursos de Educación Primaria o los primeros de Educación Secundaria. Sin embargo, todavía no hemos empezado a analizar los efectos de esos cambios. En ese contexto, el objetivo de este artículo es investigar que resultados de los estudiantes podemos esperar en este entorno tecnológico en la asignatura de Lengua Castellana y Literatura durante el $1 .{ }^{\text {er }}$ curso de Educación Secundaria Obligatoria en Cataluña (España). Para ello, hemos diseñado una investigación quasi-experimental $\mathrm{y}$ hemos registrado las metodologías de enseñanzaaprendizaje utilizadas durante dicho curso. Los resultados indican que el alumnado mejora poco su competencia comunicativa en español después de todo un curso. Todo ello nos lleva a dos conclusiones: la primera de ellas es la detección de un problema curricular, pues la mayoría de las actividades de enseñanza-aprendizaje no se orientan a la adquisición de la competencia comunicativa, sino a contenidos específicamente gramaticales o literarios; la segunda es acerca del uso de la tecnología, pues no esta no parece contribuir en exceso a mejorar esta destreza en su uso actual.
\end{abstract}

Palabras clave: Tecnología digital, educación secundaria, evaluación, aprendizaje de lenguas, TIC
Abstract
In the last three years, Spain has implemented one-to-one educational models, based on a constant and permanent use of technology in classrooms, among the last years of primary education or the first years of secondary education. However, we still have not begun analyzing 
the effects of those changes. In this context, the aim of this paper is to analyze the pupils' achievement expected within this technological environment in the subject of Spanish Language and Literature during the 1st secondary course in Catalonia (Spain). To do this, we have designed a pretest and a posttest and we have recorded those teaching-learning methods used during that course. The results bring up a discouraging picture: students poorly improved their communicative competence after a full school year. This leads us to two conclusions: the first is a curricular problem that exists, as most of the teaching-learning activities are not oriented to the communicative competence learning, instead they are oriented to grammatical or literary contents; the second one is about the current technology use, since it does not seem to contribute to improve textual skills in its current use.

Keywords: digital technologies; secondary education; assessment; language learning; ICTs

A menudo, cuando se intenta analizar los resultados del sistema educativo español en las evaluaciones internacionales en las que nuestro país participa, como miembro de la Unión Europea (UE) o de la Organización de Estados para la Cooperación y el Desarrollo Económico (OCDE), son muchas las alarmas que apuntan a deficiencias tanto en el desarrollo de las políticas educativas como en su propia concepción. Prueba de ello son los informes PISA, a cargo de la OCDE, en los que España no siempre queda en buen lugar, y las múltiples opiniones que se vierten en los medios de comunicación y en la comunidad académica acerca de ellos.

El área de Lengua Española, sin duda, es uno de los puntos candentes de todos esos informes, pues nuestros niveles de competencia comunicativa, en general, o de comprensión lectora, en particular, suelen ser especialmente llamativos, por bajos. Así, por ejemplo, lo ilustran los estudios sobre competencia comunicativa en Andalucía referidos por Fabregat (2009), en los que se certifica un nivel medio-bajo para el alumnado de Secundaria en esta competencia, con puntuaciones medias en los cursos 2005/2006 a 2007/2008 que oscilan entre el 3,2 y el 3,81 en una escala de 10. Y es que, según Sánchez Calvo (2009), nuestro alumnado -de Primaria y de Secundariapresenta carencias fundamentales en comprensión y expresión orales y escritas que, al final, acaban reflejándose en los «mediocres» resultados que obtiene nuestro Sistema Educativo, por medio de nuestros alumnos, en las periódicas evaluaciones internacionales en las que participamos.

En el fondo de la cuestión, mucho más allá del detalle de unos resultados peores de lo deseado, subyace la peculiar problemática de un área que es consciente desde hace tiempo de su necesidad de replantearse. Numerosos autores vienen señalando, de hecho, a esa cuestión, como parte del propio problema. Por un lado, está la cuestión de que la asignatura de Lengua es mucho más que una simple asignatura, pues recibe la encomienda de formar personas competentes comunicativamente (López Valero, 1998: 24), y eso es algo que supera con creces las limitaciones de los propios contenidos de la materia. Por otro lado, abunda la reivindicación de que, más allá de las exigencias del currículo, no debemos «formar lingüistas, sino hablantes», del mismo modo que no queremos «formar filólogos, sino lectores» (Mendoza y Cantero, 2003). En definitiva, en un momento en que toda esta área de conocimiento está replanteándose, son múltiples las cuestiones que debemos intentar responder, según Dolz, Gagnon y Mosquera (2009, pp. 119 y ss): 
Para la didáctica de las lenguas, las cuestiones sobre qué objetos hay que enseñar y cómo facilitar su adquisición son cruciales. ¿Qué aspecto merece privilegiarse, el de las prácticas, el de las conductas, el de los saberes tradicionales, el de los nuevos conocimientos? En relación con los alumnos, ¿qué competencias, qué capacidades deben desarrollarse? [...] Los múltiples objetivos en el ámbito de la lengua dificultan la delimita ción delos contenidos y procedimientos para enseñar y aprender. [...] ¿Cuál es el método que debe privilegiarse con quienes aprenden a leer? ¿Qué rol atribuir a los conocimientos metalingüísticos en el desarrollo de la competencia lingüística? ¿Qué gramática enseñar? ¿En qué medida el estudio de la lengua literaria puede contribuir al aumento de las capacidades lingüísticas de los alumnos?

De forma colateral a todo ello, los modelos educativos asisten a una completa revolución tecnológica que afecta a medios y modos de una forma integral. El área de Lengua Castellana, entendida como L1, no ha sido un campo especialmente abonado para la incorporación de las Tecnologías de la Información y la Comunicación (TIC), especialmente en Secundaria. Sin embargo, quizá como consecuencia de la indudable personalidad innovadora de la didáctica de las lenguas extranjeras, poco a poco las TIC también han ido incorporándose a las aulas, también en nuestra materia. Todo ello, con innovaciones que, en general, han conocido poca difusión y escasa evaluación.

En este contexto, en Cataluña se ha puesto en marcha para Secundaria un modelo «un alumno, un ordenador», con todas sus implicaciones (digitalización completa de las aulas, desaparición del libro de texto en papel, etc.); y casi de la noche a la mañana, las TIC han dejado de ser un recurso excepcional, para convertirse en el medio habitual con el que se producen los procesos de enseñanza-aprendizaje.

\section{El contexto educativo: eduCAT1x1}

A partir de cuanto acabamos de sugerir, parece necesario detenerse unos instantes para explicar, aun brevemente, cuál es el contexto educativo en el que desarrollamos nuestra investigación, el proyecto eduCAT1x1 en la Demarcación Educativa de Terres de l'Ebre, en el sur de Cataluña (España). Para empezar, sería absurdo cuestionarse siquiera la importancia de las Tecnologías de la Información y la Comunicación (TIC) en la educación para garantizar la incorporación futura del alumnado a la Sociedad del Conocimiento. Además, también queda fuera de toda duda que uno de los objetivos clave del sistema educativo español actual, en el marco de la implantación de la LOE, son sin duda las competencias. De la suma de uno y otros aspectos, se desprende la nueva concepción en la evolución del propio concepto de las TIC. No en vano, su uso ha evolucionado pasando de ser objeto de estudio y el centro del aprendizaje, desligado de las materias curriculares, en la década de los 80 , hacia posturas más propias de un completo replanteamiento sobre cómo integrarlas en el currículum, ya en la década de los 90. Y, actualmente, el interés se centra en qué contenidos y metodologías facilitan mejores aprendizajes y, evidentemente, la adquisición de las competencias, en general. Y, por supuesto, es ahí donde entra en escena la necesidad de hablar de competencia digital en el contexto de la educación obligatoria (Vivancos, 2008). Esta revolución 
conceptual, de hecho, es lo que se ha formulado por medio del cambio de terminología: de hablar de TIC, en general, hemos pasado a hablar de Tecnologías del Aprendizaje y el Conocimiento (TAC), en un intento de poner el énfasis en la aplicación específica de todas las tecnologías al contexto educativo. Es, pues, lo que Fornell y Vivancos (2010) definen como el uso de las tecnologías al servicio de una mejora en los procesos de enseñanza-aprendizaje, de evaluación y de organización. Las TIC, pues, han pasado de ser una simple herramienta, con la que renovar los modos, a constituirse en verdaderos agentes de cambio metodológico que han de permitir prácticas innovadoras y significativas; esto es, no solo hacer «de otro modo», sino hacer «más» y «mejor».

Como consecuencia de todo lo anterior, podemos afirmar sin ningún atisbo de duda que la integración de las TIC en los centros educativos es una oportunidad para innovar y gestionar un cambio. Y este cambio afecta de forma nuclear a toda la comunidad educativa y a las diferentes acciones pedagógicas: de gestión, organizativas, formativas de cada uno de los agentes integrantes (Fornell y Vivancos, 2010).

Con sus diferentes velocidades y concreciones, el programa eduCAT1x1, adaptación catalana de la estatal Escuela 2.0, tiene como punto de llegada el modelo 1x1, que le da nombre: un ordenador por alumno/a, que se erigirá en la herramienta principal de trabajo y que considera como paso necesario la sustitución de los libros tradicionales por los libros digitales. El proyecto eduCAT1x1, pues, ha significado el uso general -o casi podríamos decir masivo- de un ordenador portátil por alumno, la transformación de las aulas ordinarias en las denominadas aulas digitales, el acceso cotidiano en el contexto escolar a la red y a entornos virtuales de enseñanzaaprendizaje (EVA), con contenidos y recursos educativos en formato digital, etc.

En definitiva, este proyecto puede considerarse una concreción del proyecto Escuela 2.0 del Ministerio de Educación de España, cuya finalidad es adaptar los procesos de enseñanza y aprendizaje al siglo XXI. Hasta ahora, las nuevas tecnologías eran un apoyo esporádico o residual en la educación. A partir de ahora, deben ser parte fundamental del proceso de enseñanza y aprendizaje:

El Proyecto eduCAT1x1 no está asociado, en primera instancia, a ningún cambio metodológico específico. Son los equipos didácticos quienes deben definir el proyecto educativo de su centro, en una estrategia por fases en la que el paso del formato analógico (libro de texto, en papel) al formato digital (libro de texto digital) se hace primero, y el cambio hacia metodologías avanzadas de aprendizaje y enseñanza no debe hacerse simultáneamente, sino en los plazos que cada centro determine. El cambio metodológico es mucho más fácil una vez conectado al mundo digital». (Generalitat de Catalunya, 2011).

Aparte de innumerables cuestiones pedagógicas y organizativas (Espuny et al., 2010), es indudable que esta revolución en nuestras aulas de educación obligatoria necesita, para ser exitosa, la consolidación de una competencia fundamental, la digital, y de una gran renovación pedagógica que sea capaz de plantearse de nuevo qué enseñamos cuando enseñamos lenguas, y cuál es nuestro objetivo en esta área. Sobre lo primero, nos hemos ocupado en diferentes reflexiones anteriores (por ejemplo, 
González et al., 2012). Sobre lo segundo, centrado en la asignatura de Lengua Castellana y Literatura, vamos a profundizar a continuación.

\section{Objetivos de la investigación}

A partir del contexto que acabamos de referir, nuestro objetivo en esta investigación es analizar cómo se está aprovechando la incorporación de los microportátiles a las aulas de forma general; y, en especial, cuál es el nivel de aprovechamiento del alumnado en esta experiencia innovadora. Este análisis nos permitirá plantear propuestas encaminadas a mejorar el rendimiento académico del alumnado de esta asignatura en particular y de las demás disciplinas lingüísticas en general, así como a permitir el mayor aprovechamiento de las oportunidades que ofrece la digitalización de las aulas. Más en concreto, el objetivo de esta investigación es (1) analizar cómo la incorporación de los programas de inclusión masiva de las TIC (uno a uno, one-to-one) pueden influir en la mejora de la competencia comunicativa; y (2) comprobar si estos programas comportan el necesario cambio metodológico.

\section{Metodología}

La metodología con la que nos enfrentamos a esta investigación parte de un modelo descriptivo con paradigma mixto, cualitativo y cuantitativo, pues valoramos no solo los resultados de rendimiento académico obtenidos con los instrumentos que presentaremos a continuación, sino que también analizamos el seguimiento de las programaciones didácticas sobre las que se ha llevado a cabo la acción educativa con ese alumnado (y, especialmente, las metodologías didácticas que se han empleado con él). La generalización del modelo eduCAT1x1 (uso masivo de microportátiles) en toda la demarcación educativa nos impide el establecimiento de un grupo de control con las mismas características, pero sin el uso de microportátiles; y, por otro lado, la inexistencia de investigaciones similares a esta, con idénticos instrumentos y contextos equivalentes, no nos permite establecer comparaciones directas, motivo por el cual la investigación es más descriptiva que comparativa.

\section{Instrumentos}

El principal instrumento con el que recabamos los datos parte de los cuestionarios de comprensión lectora utilizados en 2000 por la Organización de Estados para el Desarrollo y la Cooperación (OCDE), comúnmente conocidos como «informe PISA» (Program for International Student Assessment), y cuyo detalle puede encontrarse documentado en INECSE (2005) y OCDE (2006).

Los tests de PISA de comprensión lectora parten de la idea de que esta es la capacidad de comprender, utilizar y analizar textos escritos para alcanzar los objetivos del lector, desarrollar sus conocimientos y posibilidades y participar en la sociedad (INECSE, 2005). Y a esta capacidad vienen asociándole cinco grandes procesos: la obtención de la información, la comprensión general, la elaboración de una 
interpretación, la reflexión y la valoración del contenido de un texto y la reflexión y la valoración de la forma de un texto. Los tests PISA tratan de medir dichas cinco dimensiones, tanto en textos continuos como discontinuos, con la finalidad de simular situaciones reales de lectura.

Al tratarse de procesos de comprensión lectora que deben evaluarse por medio de respuestas escritas, en muchos casos por medio de redacción elaborada, a través de las respuestas a PISA no solo podemos obtener evidencias acerca de la comprensión lectora, como las que tradicionalmente analiza la OCDE, sino también evidencias acerca de la expresión escrita.

Además, nosotros hemos adaptado uno de los textos continuos validado como prueba de comprensión lectora para convertirlo en prueba de comprensión oral, de modo que con esos dos textos -uno escrito y otro oral- hemos podido escrutar las comprensiones oral y lectora, y la expresión escrita.

Dichos textos son los siguientes, tomados de INECSE (2005):

Texto para comprensión lectora: Graffiti. Se trata de dos cartas al director de un periódico con visiones enfrentadas acerca del tópico graffiti. Sobre este texto se realizan cuatro preguntas, dos de reflexión y otras dos de interpretación. Según se recoge en INECSE (2005), es un texto especialmente cercano a los intereses de los jóvenes, motivo por el cual resulta de especial utilidad para este fin.

Texto para la comprensión oral: Gripe. Se trata de un texto informativo, en el que la comprensión se evalúa a partir de cinco preguntas: una de obtención de información, dos de reflexión, y otras dos de interpretación.

El último componente de la competencia comunicativa que nos quedaba lo hemos analizado por medio de una locución que los alumnos debían preparar y grabar ante el entrevistador, de tema genérico y un minuto de duración recomendada. Tenemos, con ello, completa la concepción clásica de Hymes (1984) de la competencia comunicativa por medio de un instrumento profusamente utilizado y validado por investigadores externos a este estudio. En la Figura 1, tratamos de reflejar esas cuatro dimensiones, junto con los test con los que las medimos: 


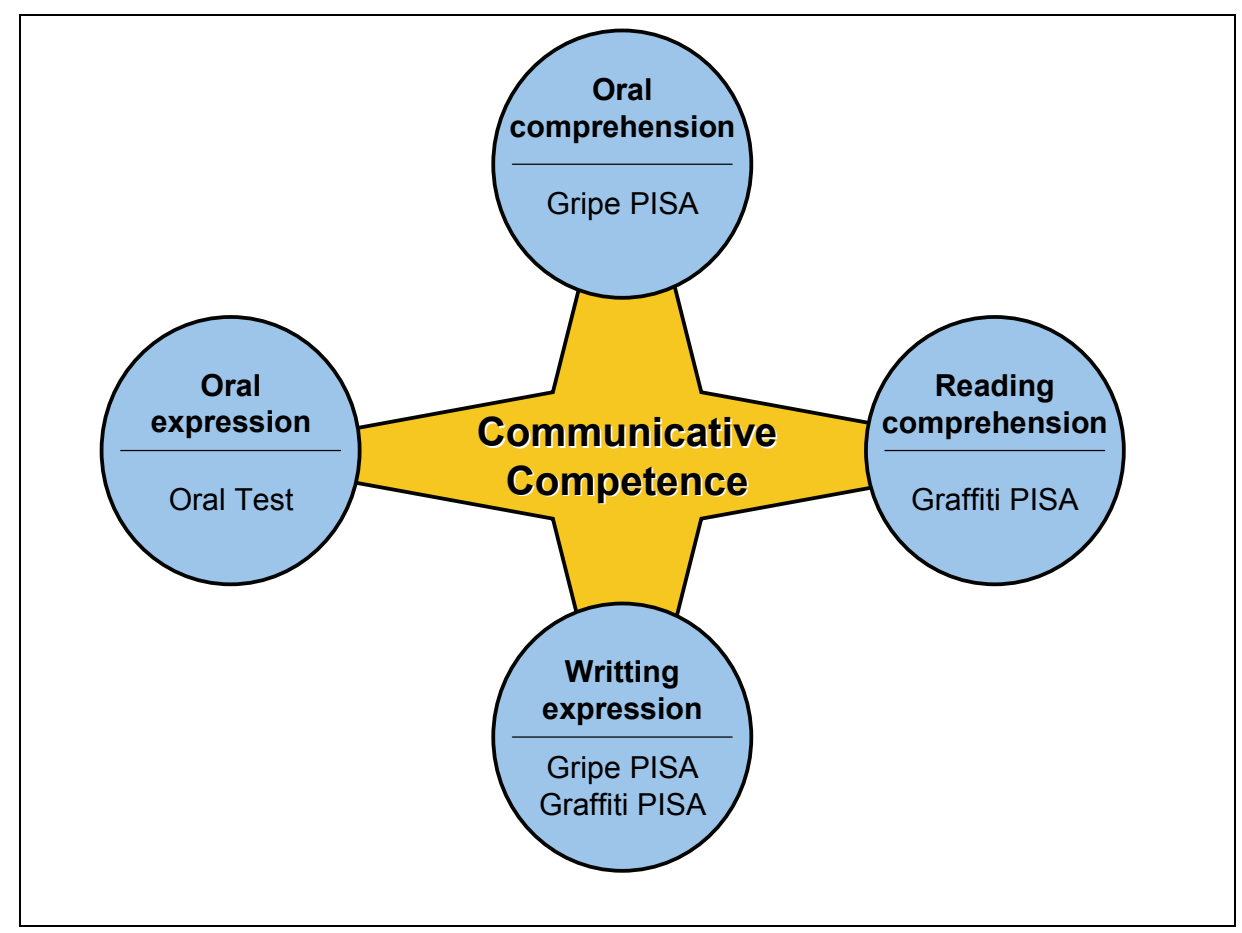

Figura 1. Competencia comunicativa.

Por medio del vaciado de las respuestas a estos tests, obtenemos un indicador general de competencia comunicativa, y cuatro subindicadores de expresión oral, expresión escrita, comprensión oral y comprensión escrita, todos ellos con un valor máximo de 1000 puntos.

De modo paralelo, y por medio de entrevistas periódicas con el profesorado que ha impartido la asignatura de lengua a los informantes de esta investigación, hemos sondeado las metodologías didácticas que han venido utilizándose a lo largo de este curso. Este sondeo se ha realizado por medio del análisis cualitativo de los diarios de clase y programaciones de aula, por medio de una tabla de registro que atendía específicamente a los tipos de actividades y al tiempo dedicado a ellas. Posteriormente, estos tipos de actividades se han ido codificando por metodologías. Una vez analizados estos datos, se han ido comentando con el profesorado mediante entrevistas que se encaminaban a confirmar los resultados de dichos análisis. 
Este cuestionario fue distribuido en pretest y postest. El pretest tuvo lugar al inicio de curso (septiembre de 2010) a todos los estudiantes 1 er $^{\text {er }}$ curso de Educación Secundaria Obligatoria (12-14 años) en los siguientes institutos públicos: INS Joaquim Bau (Tortosa), INS Les Planes (Santa Bàrbara) e INS M. Sales i Ferré (Ulldecona); todos ellos ubicados en la demarcación educativa de Terres de l'Ebre, en la provincia de Tarragona (España). El postest fue llevado a cabo a finales de mayo de 2011, al finalizar el mismo año académico, y gracias a los mismos estudiantes.

69 informantes respondieron el cuestionario $(\mathrm{N}=69)$, sobre una población de 187 individuos matriculados en ese mismo nivel educativo en los tres institutos. Representan a un universo aproximado de unos 500 individuos de estas mismas características a lo largo de toda esta demarcación educativa. La muestra final representa, pues, un $37 \%$ de la población estudiada y un $14 \%$ del universo que queremos describir.

\section{Tratamiento de los resultados}

Para finalizar con las cuestiones metodológicas, es necesario señalar que hemos procedido al análisis cuantitativo de los datos recabados utilizando los paquetes de cálculo estadístico SPSS 17.0 y Statgraphics Centurion 16. Los tests de significación se han basado en pruebas ANOVA, y siempre hemos aplicado un nivel de significación de $\mathrm{p}<0.05$. Por razones de claridad, hemos omitido los $\mathrm{p}$-valores de cada uno de los subgrupos, y las respectivas diferencias, y solo damos cuenta de ello cuando nos encontramos ante contextos de significatividad en la diferencia estadística.

\section{Resultados}

A continuación, ofrecemos los resultados obtenidos en el pretest (octubre de 2010) por el conjunto de la muestra, tanto por lo que respecta a los promedios como a las desviaciones típicas (Vid. Tabla 1):

\begin{tabular}{rcc}
\hline & Media & Desv. Típ. \\
\hline Comprensión oral & 430.04 & 214.229 \\
\hline Comprensión lectora & 409.63 & 184.828 \\
\hline Expresión oral & 527.73 & 142.770 \\
\hline Expresión escrita & 387.08 & 194.586 \\
\hline Competencia comunicativa & 438.62 & 157.019 \\
\hline
\end{tabular}

Tabla 1. Resultados del pretest. 
Y también los resultados correspondientes al postest (mayo de 2011), también con medias y desviaciones típicas (Vid. Tabla 2):

\begin{tabular}{rcc}
\hline & Media & Desv. Típ. \\
\hline Comprensión oral & 450.13 & 222.677 \\
\hline Comprensión lectora & 415.20 & 217.872 \\
\hline Expresión oral & 565.47 & 149.773 \\
\hline Expresión escrita & 407.63 & 219.495 \\
\hline Competencia comunicativa & 459.61 & 180.533 \\
\hline
\end{tabular}

Tabla 2. Resultados del postest.

Al comparar los resultados del pretest y del postest por medio de un gráfico de araña (vid. Gráfico 1), vemos que las diferencias son leves, de tal forma que las líneas azul y roja prácticamente se superponen. Además, hay que destacar que ambas se mantienen siempre por debajo del valor de 500, que no solo es la media de la escala, sino que también se corresponde con la media de comprensión lectora de la OCDE en los informes PISA (INECSE, 2005). Aparte, debemos considerar el valor correspondiente a la expresión oral, para la que no tenemos referente directo, que se sitúa por encima de la media.

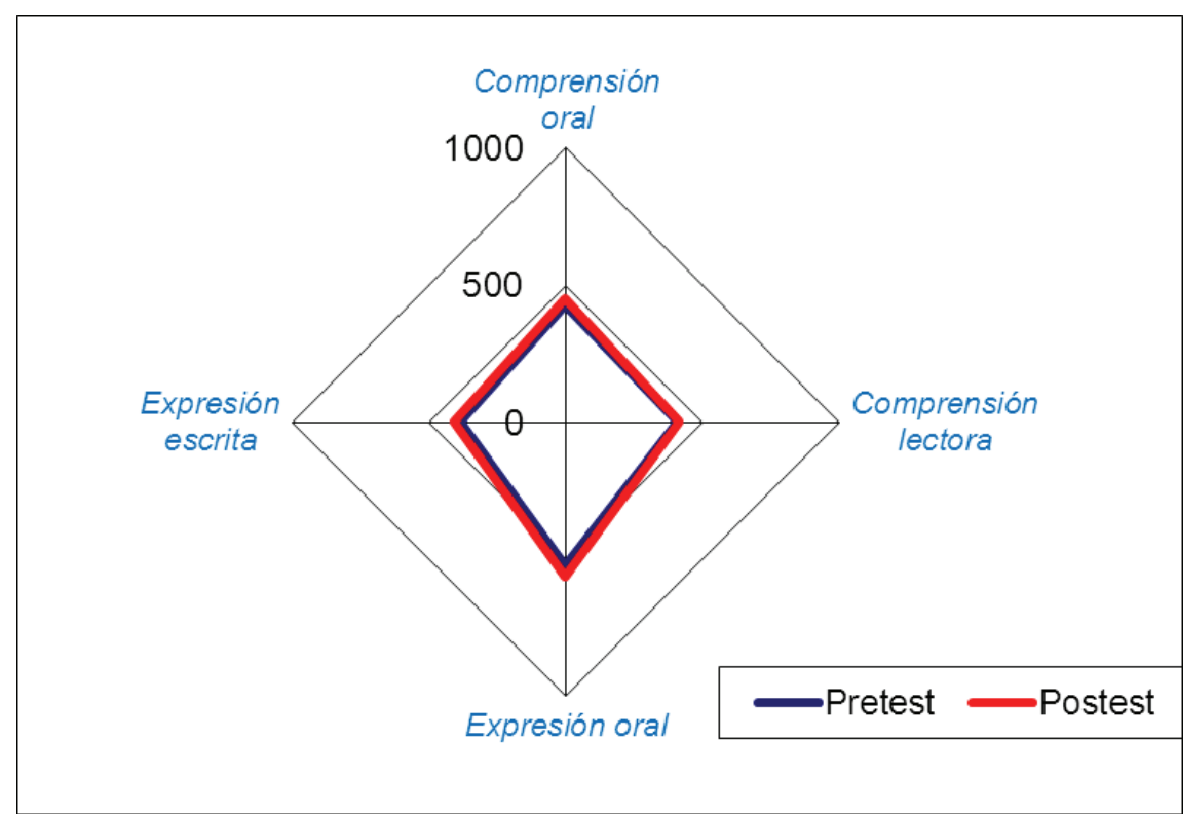

Gráfico 1. Pretest y postest. 
Como consecuencia de lo anterior, podemos analizar el aprendizaje neto de la muestra en esta experiencia (obtenido restando a los promedios del postest los resultados del pretest). Estos resultados se encuentran en la tabla

\begin{tabular}{rc}
\hline & Media \\
\hline Comprensión oral & 20.09 \\
\hline Comprensión lectora & 5.56 \\
\hline Expresión oral & 37.74 \\
\hline Expresión escrita & 20.56 \\
\hline Competencia comunicativa & 20.99 \\
\hline
\end{tabular}

Tabla 3. Aprendizaje.

Por último, y en concreto para la competencia comunicativa, ofrecemos un gráfico en el que se aprecia la leve diferencia entre los resultados del pretest y los del postest, así como el aprendizaje neto por lo que respecta a esta competencia básica (Vid. Gráfico 2).

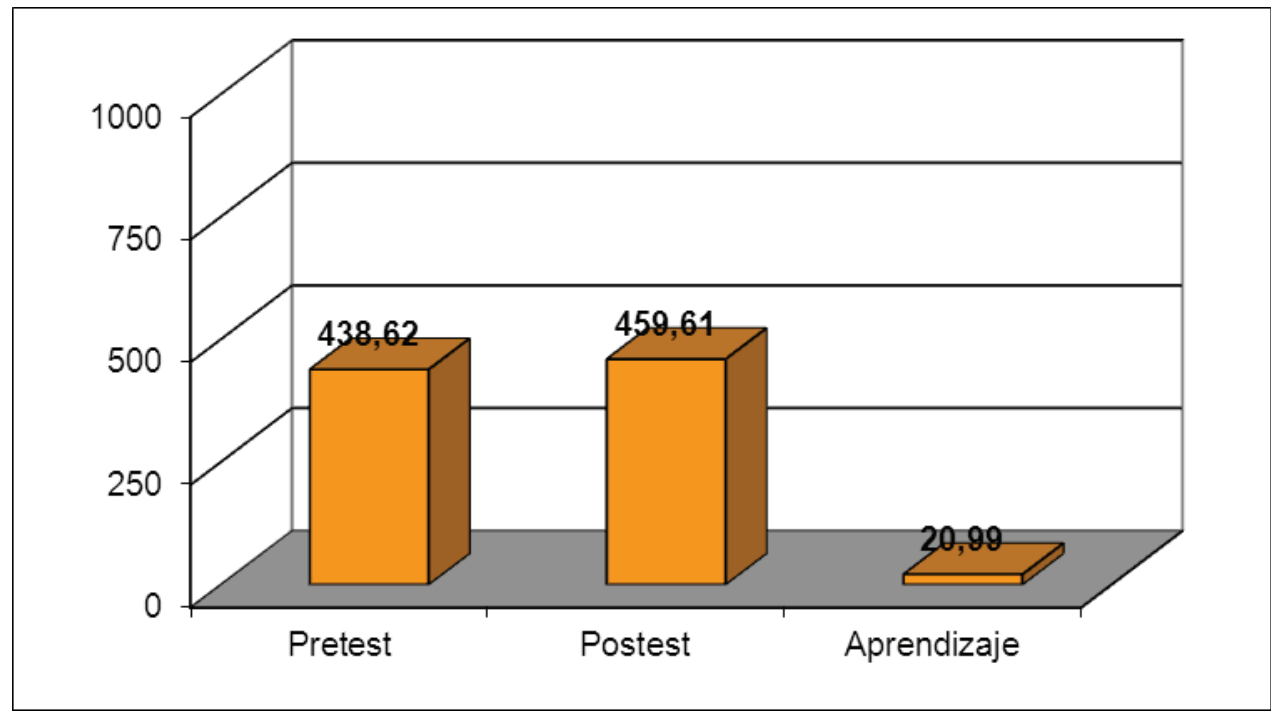

Gráfico 2. Competencia comunicativa.

En cuanto a los datos obtenidos sobre las metodologías utilizadas en el aula, las agrupamos en función de los tres objetivos de la materia enunciados: adquisición de la competencia comunicativa ( $23 \%$ de la dedicación horaria de la asignatura), por medio de actividades fundamentalmente de lectoescritura (lectura individual y grupal, resumen, comprensión lectora, etc.); contenidos y habilidades gramaticales (54 \% de la dedicación horaria), por medio de actividades desligadas de la competencia comunicativa o ligadas a ella solo de modo indirecto (conceptualización de la teoría 
gramatical; análisis gramatical, categorización léxica, habilidades gramaticales, etc.); acercamiento al hecho literario ( $23 \%$ de la dedicación horaria), por medio de reflexiones literarias teóricas, solo en algunos casos fundamentadas a partir de actividades de lectura de clásicos o textos modélicos.

\section{Discusión y análisis}

A la vista de los resultados anteriores, no hay duda de que estos merecen una contextualización evidente; tras ello deberemos reflexionar acerca de qué nos indican, y acerca de qué acciones debemos poner en marcha para mejorar la realidad del proyecto eduCAT $1 \mathrm{x} 1$ en general y de la didáctica de las lenguas en particular. Por ello, intentaremos determinar primero cuál es el nivel real de aprovechamiento y aprendizaje del alumnado estudiado, y lo pondremos en relación con el enfoque que tradicionalmente se ha venido utilizando en esta área.

Por lo que respecta a lo primero, no hay duda ninguna de que los niveles obtenidos en este test por nuestros informantes son considerablemente más bajos. Como sabemos, la media de nuestra muestra es de 438,62 puntos en el pretest y 459,61 en el postest; y, para mejor situarnos, deberemos tomar como referencia el valor de 500, que constituye la media de la OCDE. En el caso de los tests que nosotros utilizamos, España obtuvo unos resultados medios de comprensión lectora de 493 puntos, en el puesto 19 de 32 (y, como oportunamente se encargaron de destacar los medios de comunicación, por debajo del de países como Finlandia, Irlanda, Reino Unido, Suecia, Austria, Bélgica, Islandia, Noruega, Francia, Dinamarca o Suiza, aunque por encima de los resultados obtenidos por el alumnado de países como Chequia, Italia, Alemania, Hungría, Polonia, Grecia Portugal o Rusia). Es importante reconocer la diferencia de edad entre nuestro alumnado y el que habitualmente forma parte de los procesos de PISA (alrededor de 13 meses de diferencia si tomamos como punto de comparación el postest), para tomar mejor la medida de lo que los datos pueden llegar a sugerirnos. Sin embargo, las diferencias son demasiado importantes para no tomarlas en consideración; especialmente, si tenemos en cuenta que, como ventaja, nuestra muestra dispuso de dos oportunidades para realizar la misma prueba (el pretest y el postest), lo cual parece no reflejarse demasiado en los resultados finales.

Algo más parecido a nuestro caso podríamos encontrar si miramos el avance de los resultados obtenidos en la prueba de comprensión lectora más reciente, la de 2009, que inaugura un nuevo ciclo (OCDE, 2010). En esa edición del informe PISA, los resultados de España en comprensión lectora se empobrecieron aún más, de tal modo que nuestra media bajó de los 475 puntos y nos sitúa en el grupo de los países cuya diferencia con la media de la OCDE es estadísticamente significativa (de nuevo, junto con países como Grecia e Italia).

Como último apunte a esta reflexión, debemos señalar que, en los informes del INECSE (2005), se intentaba señalar que nuestro alumnado era muy homogéneo, como 
resultado de un sistema educativo universal, público y equitativo, con un gran afán por la cohesión social. Como prueba de ello se apuntaba a la baja desviación típica en los resultados, con valores ahora sí por debajo de la media, en torno 85 puntos. En nuestro caso, por el contrario, no podemos decir lo mismo, pues los valores de desviación típica no solo son casi el doble; sino que, además, se incrementan en el postest con respecto del pretest, como prueba de que nuestro sistema no es tan equitativo en sus resultados como parecía apuntar el informe del INECSE (2005).

Así, lo que nos encontramos es un alumnado con graves carencias por lo que respecta a su competencia comunicativa que, además, consigue paliarlas tras un año de trabajo en la materia en un grado muy bajo. Ciertamente, los 21 puntos de media que «mejora» la muestra parecen muy escasos en una escala de 1000 puntos; y eso es especialmente grave cuando nos planteamos que el conjunto de los informantes se sitúa por debajo del umbral medio de la $\operatorname{OCDE}$ (y, por otro lado, en un nivel que consideraríamos «suspenso» en una valoración académica tradicional). Hasta donde conocemos, no hay investigaciones que hayan utilizado estos mismos tests del modo que nosotros, al inicio y al final de un año académico; y por ello no podemos establecer una comparación directa. Pero la experiencia docente se encarga sobradamente de sugerirnos que se trata de un resultado demasiado pobre para lo que debería ser.

Según el citado informe, el diagnóstico de PISA en comprensión lectora es muy revelador de la naturaleza de nuestro alumnado:

1. Nuestro alumnado no presenta excesivos problemas al opinar, interpretar o inferir a partir de cuanto lee, pero esa lectura acaso sea a menudo superficial, de tal modo que la falta de una lectura rigurosa que le impide recabar el dato $\mathrm{u}$ obtener la información precisa en un texto.

2. Con carácter general, en cualquier nivel de comprensión, el alumnado tiene más dificultades para entender un texto narrativo que expositivo. En nuestro caso, ninguno de los textos se ajusta a este apunte, y aun así son constatables los problemas de comprensión.

3. También parece que las alumnas y los alumnos españoles tienen alguna dificultad para comprender textos discontinuos: diagramas, gráficos, tablas, esquemas, etc. muy necesarios para resolver problemas de otras áreas de conocimiento. Salvando las distancias, acaso eso se materialice en la prueba de comprensión oral.

4. Tal y como se sugiere en nuestros datos (que no hemos expuesto por limitaciones de espacio), tanto los informes PISA como el resto de las evaluaciones nacionales llevadas a cabo desde el INECSE ponen de manifiesto que las alumnas obtienen unos resultados en comprensión lectora significativamente mejores que los logrados por los alumnos. 
La gravedad de este bajo nivel en una competencia básica de este calado está fuera de toda duda. Y así, podemos tomar como pauta el diagnóstico de Fabregat (2009). En su análisis del nivel de competencia comunicativa del alumnado andaluz de ESO, obtiene resultados que rondan también los 450 puntos; con carencias importantes especialmente en expresión escrita. Y es que, en una escala en la que el valor medio es 500, los alumnos con valores inferiores a ese nivel tendrán serias dificultades a la hora de aprobar las asignaturas más directamente conectadas con las habilidades que tienen que ver con la lectura eficiente y con la escritura académica (Fabregat, 2009, p. 25). En nuestro caso, esto es especialmente relevante si tenemos en cuenta que, de las cuatro subcompetencias, son precisamente las escritas (comprensión lectora y expresión escrita) las que peores niveles presentan de inicio en el pretest, y las que menos consiguen verse mejoradas. Y no hay duda de su importancia en los procesos más habituales de estudio. Volvemos, pues, de nuevo, a topar directamente con la necesidad de tomar conciencia de la importancia de las materias denominadas instrumentales por su contribución a la construcción del conocimiento y, por tanto, al aprendizaje.

Llegados a este punto, es justo encajar estos datos en función de cuáles son los procedimientos didácticos utilizados en el aula. Y así, veremos que los docentes que han trabajado con este alumnado, en plena sintonía con el conjunto de docentes del área, han atacado de un modo tradicional la docencia de la materia, utilizando a su servicio los recursos tecnológicos a su alcance (pero innovando escasamente en la concepción global de su acción docente). Esto quiere decir que en más de tres cuartas partes de la dedicación horaria de la materia, el alumnado se ha dedicado a tareas que solo indirectamente se relacionan con la necesidad de mejorar la competencia comunicativa (como, por ejemplo, las relacionadas con el conocimiento de la lengua en cuanto que sistema, pero desvinculadas del uso eficiente); o a tareas que, aun directamente relacionadas con ese aprendizaje, no están situadas en una secuencia didáctica que les confiera sentido global (esto es, por ejemplo, ejercicios de ortografía de las letras que después no cuajan en técnicas de redacción entendida como tal).

Todo ello, por supuesto, se prodiga en el nuevo contexto tecnológico que caracteriza el proyecto eduCAT1x1. Y así, los docentes han adaptado los modos clásicos de enseñar lengua a los nuevos recursos. En muchos casos, lo único que ha cambiado ha sido el medio, la herramienta (de libros en papel, a libros digitales; de pizarras tradicionales a pizarras digitales interactivas); en otros casos, los procesos siguen siendo exactamente los mismos, y con los mismos soportes (libreta, bolígrafo, etc.). La tecnología, además, permite realizar hasta la saciedad tareas que antes resultaban tediosas y que ahora parecen incluso atractivas para el alumnado: rellenar huecos, completar secuencias, relacionar conceptos, etc.

Llegados a este punto, no podemos dejar de constatar una paradoja especialmente importante: las pruebas que hemos realizado al alumnado están encaminadas a medir exclusivamente su nivel de competencia comunicativa, cuando eso no es lo que ocupa la mayor parte de su tiempo de aprendizaje. La mejora de la competencia comunicativa es, a decir de cualquier docente de lenguas, el objetivo último y casi sagrado de nuestra 
área. Sin embargo, y allí está la paradoja: ¿por qué no se le dedican los más de los esfuerzos? Esto es, si el objetivo es mejorar las competencias comunicativas, ¿por qué este objetivo consume directamente tan poco tiempo del docente y del discente?

La normativa española y catalana en que se fijan los currículos de las asignaturas es clara a este respecto. Y, como muestra de ello, citaremos algunos ejemplos exentos de duda: el Ministerio de Educación y Ciencia (1991) ya señalaba que «el objetivo de la Lengua y la Literatura en la formación del alumnado de Secundaria debe ser progresar en las cuatro habilidades comunicativas básicas: hablar, escuchar, leer y escribir». En palabras de López Valero (1998, p. 26), el objetivo debe ser favorecer en el alumnado el dominio de todos los recursos de su lengua para expresarse y comunicarse en cualquier situación que la vida le plantee; es decir, el desarrollo de la competencia comunicativa en su concepción clásica (Hymes, 1984). Más adelante, el artículo 22 de la Ley Orgánica de Educación (MEC, 2006), dentro de los objetivos y finalidades de la Educación Secundaria Obligatoria (ESO), reconoce que uno de ellos es «comprender y expresar con corrección, oralmente y por escrito, en la lengua castellana y, si la hubiere, en la lengua cooficial de la Comunidad Autónoma, textos y mensajes complejos, e iniciarse en el conocimiento, la lectura y el estudio de la literatura». Lo mismo determina la autoridad educativa regional (en este caso, el Departament d'Educació de la Generalitat de Catalunya) en el decreto donde se regulan los currículos actualmente vigentes. Dicho decreto, además de fijar la comunicativa como competencia básica, dispone que «en la Educación Secundaria Obligatoria debe plantearse el desarrollo integral y armónico de los aspectos intelectuales, afectivos y sociales de la personal, entre los cuales la educación lingüística y comunicativa ocupa un lugar preferente. Hay que formar hablantes plurilingües e interculturales; la consolidación de la competencia plena en catalán, la lengua propia de Cataluña, y en castellano es la garantía de que la escuela proporciona al alumnado la competencia que necesita para tener idénticas oportunidades. Esta competencia plurilingüe e intercultural incluye el respeto por la diversidad lingüística y el deseo de aprender otras lenguas y de aprender todas las lenguas y culturas, de un modo totalmente integrado, el afianzamiento del dominio de las competencias comunicativas audiovisuales y digitales necesarias para ser competente en nuestra sociedad». Y, ya para terminar, la Llei d'Educació de Catalunya (Generalitat de Catalunya, 2009) fija en su artículo 59 que una de las aspiraciones de la ESO es precisamente que el alumnado «desarrolle en el nivel adecuado, como forma de conocimiento reflexivo, de formación de pensamiento y de expresión de ideas, las habilidades y competencias culturales, personales y sociales relativas a [...] la expresión y comprensión orales, la expresión escrita y comprensión lectora, las competencias matemáticas y las competencias necesarias para el uso de las nuevas tecnologías y de la comunicación audiovisual». Parece, pues, que no hay duda de la preeminencia de ese objetivo didáctico...

Sin embargo, la práctica docente es otra, motivo por el cual no pocas voces -a las que nos sumamos- reivindican la necesidad de replantear la materia en general y el currículo en particular. $\mathrm{Y}$ es que algo falla en la tradicional transposición didáctica 
(Chevallard, 1985) de los saberes en lenguas, que no solo deben enfocarse hacia los conocimientos gramaticales y literarios en particular, sino especialmente a las prácticas lingüísticas de referencia (Bronckart \& Plazaola, 1998; Marschall et al., 2000).

Así, por ejemplo, podemos partir de las tres finalidades de la didáctica de las lenguas, según Dolz, Gagnon y Mosquera (2009). La primera finalidad comprende habilidades tales como hablar, escuchar, leer, escribir e interactuar (Nonnon, 2000; Garcia-Debanc \& Plane, 2004; Segovia, 2012); la segunda apunta a la reflexión sobre el funcionamiento de la comunicación, sobre el sistema y sobre el uso de la lengua (Coste, 1985; Dolz \& Meyer, 1998) y garantiza un dominio consciente de los comportamientos verbales; y la tercera se caracteriza por el constante retorno a los textos literarios (Rosier, 2002), pero también a los conocimientos ligados con la historia, los usos, las normas y el patrimonio de la lengua. Tras la reflexión anterior, acerca de la práctica de los grupos evaluados, es fácil convenir que la mayor parte del tiempo de aprendizaje se dedica a la segunda y a la tercera finalidades. Y eso es especialmente grave en tanto que se obvia la primera de ellas y la segunda, que debería estar siempre subordinada a la primera, se emancipa de su servicio a la adquisición de la competencia comunicativa, sin la que carece de sentido.

La presión de las autoridades por revertir esta situación, como decíamos, no es nueva, hasta el punto de que ya en 2000 el Marco Común Europeo de Referencia para las Lenguas, publicado por el Consejo de Europa, abogaba por la necesidad de este cambio en un contexto plurilingüe en el que la formación lingüística debe orientarse netamente hacia la práctica.

La transposición didáctica en las áreas de lenguas debe enfocarse hacia lo comunicativo, según Batanaz (2003, p. 272), pues es la mejor forma de lograr que el proceso de enseñanza-aprendizaje se ponga al servicio de los objetivos propuestos, especialmente aquellos que tienen que ver con la primera finalidad de Dolz, Gagnon y Mosquera (2009): hablar, escuchar, leer, escribir e interactuar.

Así pues, sin duda, esa paradoja de la que hablábamos no se ha resuelto en el proyecto eduCAT1x1. Cabría pensar, pues, si podría haberse resuelto, en un contexto en el que se ha cambiado el medio, se han ampliado los recursos, y no se ha abogado por una reflexión profunda en el seno del área y por un meditado cambio en la metodología. Y es que desde hace años viene recomendándose desde el área de Didáctica de las Lenguas la importancia de utilizar en la formación de la lengua materna un enfoque comunicativo-funcional, ya que este permite trabajar en el aula «con la perspectiva de avanzar en el conocimiento y mejora de la acción discursiva» (López Valero y Encabo Fernández, 2002). Se trata de perfeccionar, en definitiva, la práctica del lenguaje, ya sea por este medio o por medio de procedimientos más textuales (Lomas y Osoro, 1997, p. 23); pues solo de este modo, desarrollando verdaderas competencias, podremos permitirle al alumno «aplicar, concretar el conocimiento en el entorno sociocultural, laboral y en otros contextos académicos» (Escamilla, 2008), circunstancia que ya se ve que el modelo actual no consigue, a tenor de los resultados recogidos en esta investigación. 
Nuestro sistema, en su versión tradicional, o ya en su forma más tecnologizada, parece no participar del afán real de fomentar en el alumnado una competencia comunicativa entendida como un compendio de conocimientos de la lengua, habilidades que tienen que ver con la comprensión lectora o la composición de textos orales y escritos, «por no hablar de aquellas destrezas [lingüísticas y comunicativas] que favorecen la participación en la vida social» (Pérez Esteve y Zayas, 2007, p. 7). Y, por tanto, para que los resultados en tests como que el hemos llevado a cabo mejoren, es prescriptiva «la utilización de la lengua en diferentes contextos, en situaciones comunicativas diversas, y como instrumento de comunicación oral, escrita, de aprendizaje y de socialización» (Horch, 2008). Y eso es algo que el modo «clásico» de enseñar lengua en España no lleva a término; y que eduCAT1x1, sin duda, no solo no resuelve, sino que perpetúa. Y ello es así porque al enseñar lengua no partimos de la idea de que una actuación competente siempre implica el uso de conocimientos interrelacionados con habilidades y actitudes (Zabala y Arnau, 2007); y, en la medida en que el conjunto de contenidos trabajados en el aula no se orientan específicamente a esta finalidad, difícilmente podrá conseguirse que el alumnado produzca textos -orales y escritos- que se ajusten a situaciones comunicativas reales o simuladas, finalidad última de la materia (y, por otro lado, uno de los aspectos que intenta medir PISA en sus tests de comprensión lectora).

Hasta cierto punto, un aspecto que podríamos plantearnos a continuación es quién tiene la responsabilidad en esta situación de bajo aprovechamiento académico, y en qué medida de esa responsabilidad participa la innovación tecnológica inherente a eduCAT1x1. Sin duda, hay que buscar la causa del problema, entre otros lugares, en la excesiva parcelación de los contenidos y saberes -modernamente, de las competencias- en la ESO, pues eso ha contribuido a unir indisolublemente el aprendizaje de la competencia lingüística o comunicativa y la materia de Lengua (Fabregat, 2009). Si bien el objetivo principal de la materia es la consolidación de la competencia, no hay duda de que una vez más debemos suscribir aquello de que «todos los docentes lo somos de lenguas», reinvindicación clásica del profesorado de esta área, que se siente solo frente a un reto que, de suyo, debemos asumir todos los miembros del claustro. A ello, por supuesto, debemos añadir otros aspectos, como la necesidad ya mencionada de replantear el currículo y atender al verdadero objetivo de la materia, compartido por supuesto por los demás.

En ello, por último, eduCAT1x1 no es en absoluto el responsable del «fracaso» de unos esfuerzos que no han rendido lo suficiente. Sin embargo, no hay duda tampoco de que la escasa planificación de este cambio hacia la tecnología como recurso general (González et al., 2012) no contribuye demasiado a la mejora de la situación; pues, al final, estamos vertiendo vino viejo en odres nuevos, no renovando los modos para que sean más eficaces y para que aumenten el aprendizaje. En su versión más ramplona, eduCAT1x1 fuerza al alumnado a repetir una y otra vez las estrategias fill-in-the-gaps; y eso, que desde luego no la es causa principal del bajo rendimiento detectado, tampoco contribuye a resolverlo. En su condición de recurso, o de medio, pues, 
eduCAT1x1 difícilmente podría resolver un problema de tan hondo calado como el de las carencias en lectoescritura; pero, nuevamente, hay que reconocer que puedo ayudar a perpetuarlas, al dejar el profesorado la idea de que está innovando para mejorar, cuando en realidad está innovando por innovar.

De todo ello se desprenden cuatro grandes conclusiones provisionales que remarcamos a continuación, como corolario de todo lo referido hasta el momento en este epígrafe final: (1) el alumnado de secundaria analizado, como se apunta en el informe PISA, presenta problemas importantes en comprensión lectora que no deben ser desatendidos; (2) la inclusión tecnológica masiva, por sí sola, no resuelve mejora el aprendizaje; indudablemente, supone un aumento de la motivación en primera instancia, pero ello no determina mejores niveles de adquisición de las competencias; (3) el cambio tecnológico operado no ha conllevado un necesario cambio metodológico, sino un cambio exclusivo de instrumentos de trabajo, como atestigua la escasa modificación de las programaciones; y (4) en la materia de Lengua Castellana y Literatura sigue sin cambiarse a un paradigma de trabajo por competencias, que atacaría de modo frontal el problema inicial confirmado en la primera conclusión.

Para finalizar, y a nuestro modo de ver, debemos ofrecer unas pinceladas de cuál es el camino para mejorar los resultados, lo que consideraríamos unas generales «propuestas de mejora». Como se deducirá de lo anterior, parte de la solución es que todos asumamos que la competencia lingüística o comunicativa es transversal y, por tanto, la deslocalicemos del área de Lengua, en este caso. Es imprescindible que ejerzamos entre todos una presión constante sobre los docentes de las demás disciplinas para que contribuyan a la adquisición de esta competencia (Chartrand \& Blaser, 2006). Como recomienda Fabregat (2009), es preciso arraigar en los claustros el concepto de escritura a través del currículo, de tal modo que la creación de textos sea el eje instrumental fundamental, con independencia de cuál sea el contenido de dicho texto y en qué materia eso se trabaja, en sintonía con las propuestas de Cassany (1988, 1993, 1995, 1999, 2006). En ellas, se enfoca el aprendizaje de la escritura como una tarea compleja que requiere atención en sí misma, y a la cual se subordinan conceptos y procedimientos que anteriormente se erigían como objetivos autónomos (especialmente, los contenidos gramaticales).

Por su parte, eso nos lleva a la segunda de las acciones que debemos llevar a término, la renovación metodológica del área. Debemos tomar conciencia de cuáles son los verdaderos objetivos de la materia y los medios de los que disponemos (tecnológicos o no); y todo ello, en nuestro contexto del proyecto eduCAT1x1, sin olvidar nunca que la tecnología nunca es el fin sino el medio, como ocurre demasiado a menudo al hablar de tecnología educativa. 


\section{Referencias bibliográficas}

BATANAZ, L. (2003). Organización escolar. Bases científicas para el desarrollo de las Instituciones educativas. Córdoba: Servicio de Publicaciones de la Universidad de Córdoba.

BRONCKART, J.-P.; PLazaola, I. (1998). La transposition didactique. Histoire et perspectives d'une problématique fondatrice, Pratiques, 97-98, 35-58.

CASSANY, D. (1988). Describir el escribir. Barcelona: Paidós, Paidós Comunicación, 37

CASSANY, D. (1993). Reparar la escritura: didáctica de la corrección de lo escrito. Barcelona: Graó.

CASSANY, D. (1995). La cocina de la escritura. Barcelona: Anagrama.

CASSANY, D. (1999). Construir la escritura. Barcelona: Paidós, Papeles de pedagogía, 42.

CASSANY, D. (2006). Taller de textos. Barcelona: Paidós, Papeles de pedagogía, 68.

CHARTRAND, S. G.; BLASER, C. (2006). Fonction epistemique des genres disciplinaires scolaires: prolegomenes a un champ de recherches. Schneuwly B. \& Thevenaz-Christen, T. (Eds.). Le travail de l'enseignant et l'objet enseigne. Le cas du francais langue premiere. Bruxelles: De Boeck. 179-194.

CHEVALLARD, Y. (1985). La transposition didactique; du savoir savant au savoir enseigné. Paris: La Pensée Sauvage.

COSTE, D. (1985). Métalangages, activité métalinguistique et enseignement/apprentissage d'une langue étrangère. $D R L A V, 32,63-92$.

DOLZ, J.; GAGNON, R.; MOSQUERA, S. (2009). Didáctica de las lenguas. Una disciplina en proceso de construcción. Didáctica. Lengua y Literatura, 21-117-141.

DOLZ, J.; MEYER, J.-C. (1998). Activités métalangagières et enseignement du français. Actes des journées d'études en didactique du français. Berna: Peter Lang.

ESCAMILLA, A. (2008). Las competencias básicas. Claves y propuestas para su desarrollo en los centros. Barcelona: Graó.

ESPUNY, C.; GISBERT, M.; GONZÁLEZ, J.; COIDURAS, J. (2010). Los seminarios TAC. Un reto de formación para asegurar la dinamización de las TAC en las escuelas. Edutec, 34. 1-20.

FABREGAT, S. (2009). Competencia lingüística y expresión escrita en ESO: cinco textos de Daniel Cassany. Caleidoscopio, revista de contenidos educativos del CEP de Jaén, 2. 57-66.

FORNELL, R.; VIVANCOS, J. (2010). Educació el pla TAC de centre. Col-lecció $T A C$-1. Departament d'Educació. Generalitat de Catalunya. 
GARCIA-DEBANC, C.; PLANE, S. (2004). Comment enseigner l'oral à l'école primaire? Paris: Hatier.

GENERALITAT DE CATALUNYA (2007). DECRET 143/2007, de 26 de juny, pel qual s'estableix l'ordenació dels ensenyaments de l'educació secundaria obligatòria. Barcelona: Generalitat de Catalunya. Disponible en: www.gencat.cat/educacio.

GENERALITAT DE CATALUNYA (2009): Ley 12/2009, de 10 de julio, de Educación. Barcelona: Generalitat de Catalunya. Quaderns de Legislació, 82. Disponible en: http://www.gencat.cat/educació.

GENERALITAT DE CATALUNYA (2011). EduCAT1x1 (2011). Generalitat de Catalunya.

GONZÁLEZ, J.; ESPUNY, C.; DE CID, M. J.; GISBERT, M. (2012). INCOTIC-ESO. Cómo autoevaluar y diagnosticar la competencia digital en la Escuela 2.0. Revista de Investigación Educativa, 30. 287-302.

HORCH, M. (2008). Educar en competencias. Cuadernos de Pedagogía, 376. Barcelona.

HYMES, D. H. (1984). Vers la competénce de Communication. Paris: Hatier.

INECSE (2005). Programa PISA. Pruebas de comprensión lectora. Madrid: Ministerio de Educación y Cultura.

LOMAS, C. (1999). Cómo enseñar a hacer cosas con las palabras. Barcelona: Paidós.

LÓPEZ VALERO, A. (1998). La nueva configuración en la enseñanza de la Lengua y la Literatura (Educación Secundaria). Revista Interuniversitaria de Formación de Profesorado, 31. 22-33.

LÓPEZ VALERO, A.; Encabo Fernández, E. (2002). Introducción a la didáctica de la Lengua y la Literatura. Un enfoque sociocrítico. Barcelona: Octaedro-EUB.

MARSCHALL, M. et. al. (2000). La transposition didactique des notions énonciatives dans les manuels d'enseignement des langues vivantes. Friburgo: Editions Universitaires.

MEC (1991). Real Decreto 1345/1991, de 6 de septiembre, por el que se establece el currículo de la Educación Secundaria Obligatoria. Madrid: BOE, 13/09/1991.

MEC (2006). Ley Orgánica 2/2006, de 3 de mayo, de Educación. Madrid: Ministerio de Educación.

MENDOZA, A.; CANTERO, F. (2003). Didáctica de la Lengua y la Literatura: aspectos epistemológicos. Mendoza, A. (coord.). Didáctica de la Lengua y la Literatura. Madrid: Prentice Hall. 
NONNON, E. (2000). L'enseignement de l'oral et les interactions verbales en classe : champs de référence et problématiques. Revue française de pédagogie, 129, 89-103.

OCDE (2006). PISA 2006. Marco de la evaluación. Madrid: Santillana Educación.

PÉREZ ESTEVE, P.; Zayas, F. (2007). Competencia en comunicación lingüística. cMadrid: Alianza Editorial.

ROSIER, J.-M. (2002). La didactique du français. Que sais-je? Paris: PUF

SÁNCHEZ CALVO, A. (2009). La lengua en las aulas. Revista Iberoamericana de Educación, 49/2. 1-8.

SEGOVIA, B. (2012). La adquisición de la competencia narrativa a través del cómic en la Escuela Primaria. Revista Complutense de Educación, 23(2), 375-399.

VIVANCOS, J. (2008). Tratamiento de la información y competencia digitales». Madrid: Alianza.

ZABALA, A.; Arnau, L. (2007). Cómo aprender y enseñar competencias. Barcelona: Graó.

\section{Correspondencia con los autores}

Juan GONZÁLEZ MARTÍNEZ

Universitat Rovira i Virgili

Campus Terres de l'Ebre

Avda. de Remolins, 13-15

43500 Tortosa (Tarragona, España)

e-mail: juan.gonzalezm@urv.cat 